\title{
Efficacy of sulphadoxine-pyrimethamine with or without artesunate for the treatment of uncomplicated Plasmodium falciparum malaria in southern Mozambique: a randomized controlled trial
}

\author{
Elizabeth N Allen1, Francesca Little ${ }^{2}$, Tunisio Camba ${ }^{3}$, Yasmin Cassam³, \\ Jaishree Raman ${ }^{4}$, Andrew Boulle ${ }^{5}$ and Karen I Barnes*1
}

Address: ${ }^{1}$ Division of Clinical Pharmacology, Department of Medicine, University of Cape Town, South Africa, ${ }^{2}$ Department of Statistical Sciences, University of Cape Town, South Africa, ${ }^{3}$ Ministry of Health, Mozambique, ${ }^{4}$ Malaria Research Lead Programme, Medical Research Council, Durban, South Africa and ${ }^{5}$ School of Public Health and Family Medicine, University of Cape Town, South Africa

Email: Elizabeth N Allen - elizabeth.allen@uct.ac.za; Francesca Little - francesca.little@uct.ac.za; Tunisio Camba - tmcamba@gmail.com; Yasmin Cassam - yascassam@gmail.com; Jaishree Raman - jaishree.raman@mrc.ac.za; Andrew Boulle - andrew.boulle@uct.ac.za;

Karen I Barnes* - karen.barnes@uct.ac.za

* Corresponding author

Published: 26 June 2009

Malaria Journal 2009, 8:141 doi:10.1186/1475-2875-8-141
Received: 21 January 2009

Accepted: 26 June 2009

This article is available from: http://www.malariajournal.com/content/8/I//4I

(C) 2009 Allen et al; licensee BioMed Central Ltd.

This is an Open Access article distributed under the terms of the Creative Commons Attribution License (http://creativecommons.org/licenses/by/2.0), which permits unrestricted use, distribution, and reproduction in any medium, provided the original work is properly cited.

\begin{abstract}
Background: An artemisinin-based combination therapy, artesunate (AS) plus sulphadoxinepyrimethamine (SP), was compared to SP monotherapy to provide evidence of further treatment options in southern Mozambique.

Methods: Between 2003 and 2005, 4 II patients over one year and $10 \mathrm{~kg}$ with uncomplicated Plasmodium falciparum malaria were randomly allocated SP (25/I.25 mg per $\mathrm{kg}$ day 0 ) or AS/SP (as above plus $4 \mathrm{mg} / \mathrm{kg}$ artesunate days 0,1 and 2). Allocation was concealed, but treatment was open-label except to microscopists. The primary objective was the relative risk of treatment failure, which was assessed using World Health Organization response definitions modified to a 42-day follow-up.

Results: Of the 4 II subjects enrolled, $359(87.3 \%)$ completed the follow up period (SP $n=175, A S / S P n$ $=184)$. A survival analysis including 408 subjects showed that the polymerase chain reaction-adjusted cure rates were $90.4 \%$ (95\% confidence interval [Cl] 84.9\%-93.9\%) and $98.0 \%(95 \% \mathrm{Cl} 94.8 \%-99.3 \%)$ for SP and $\mathrm{AS} / \mathrm{SP}$ respectively. Multivariable analysis showed that treatment with AS/SP decreased the relative hazard of treatment failure by $80 \%$ compared to SP (hazard ratio [HR] 0.2; $95 \% \mathrm{Cl} 0.1-0.6$ ) and age over seven years decreased the relative hazard of failure by $70 \%(\mathrm{HR} 0.3 ; 95 \% \mathrm{Cl} 0.1-0.9)$, when compared to younger age. However, having a quintuple $\mathrm{dhfr} / \mathrm{dhps}$ mutation increased the relative hazard of failure compared to fewer mutations (HR 3.2; 95\% Cl I.3-7.5) and baseline axillary temperature increased the relative hazard of failure by $50 \%$ for each ${ }^{\circ} \mathrm{C}$ increase (HR I.5; $95 \% \mathrm{Cl} \mathrm{I.I-2.2).}$

Conclusion: While both treatments were efficacious, AS plus SP significantly decreased the relative hazard of treatment failure compared to SP monotherapy Artesunate plus sulphadoxine-pyrimethamine, but not sulphadoxine-pyrimethamine monotherapy, met the current WHO criteria of $>95 \%$ efficacy for policy implementation.
\end{abstract}

Trial registration: NCT00203736 and NCT002038I4 


\section{Background}

Mozambique (population circa 20,000,000) is a poor country with a life expectancy of 45 years. Malaria is responsible for a large health burden, including an estimated $19 \%$ of deaths among children under five years of age [1]. Transmission intensity of the predominantly Plasmodium falciparum malaria varies throughout the country according to the season, other environmental factors, and the use of vector control measures. A treatment policy with effective anti-malarials is a key component of malaria control programmes and data regarding resistance of parasites to anti-malarial drugs are now a critical factor in drug policy decision-making. While chloroquine was the national policy in Mozambique for the treatment of uncomplicated malaria as recently as 2003, evidence of its poor efficacy throughout Africa since the 1980s signalled a need for alternative anti-malarials [2]. Artemisinin-based combination therapy (ACT) is now generally considered the best treatment for uncomplicated falciparum malaria provided the partner drug is efficacious. ACT deliverers a more rapid cure compared to non-ACT, reducing gametocyte carriage (the parasites' sexual stage, thereby reducing infectivity), and delaying the development of anti-malarial resistance [3-5]. The artemisinin derivative, artesunate (AS), is particularly effective in the rapid treatment of uncomplicated malaria, and its use in combination with the longer-acting sulphadoxinepyrimethamine (SP) is currently one of four forms of ACT recommended by the World Health Organization (WHO) $[6,7]$. From a programmatic perspective, AS plus SP has the unique advantage of the full dose of the partner drug being administered under supervision when malaria is diagnosed.

There was clear evidence that ACTs substantially reduced treatment failure, recrudescence and gametocyte carriage but this had not been reported for Mozambique [8]. However, amodiaquine plus SP was selected as the national treatment policy for uncomplicated falciparum malaria due to its lower cost. At this time the Ministry of Health recommended that a phased implementation of an ACT be studied in parallel in Maputo Province. Efficacy of SP monotherapy had been assessed by the South East African Combination Anti-malarial Treatment (SEACAT) evaluation in Namaacha and Matatuine Districts, Maputo Province, during 2002 and data showed that a combined polymerase chain reaction (PCR)-adjusted cure rate exceeded the $80 \%$ level considered for it to be combined with other anti-malarials to sustain its useful therapeutic life $[7,9]$. The prevalence of the quintuple dihydrofolate reductase (dhfr) and dihydropteroate synthetase (dhps), mutation across all study sites was 5-6\% throughout the study period [[10], Raman J personal communication]. This randomised controlled trial (RCT) was conducted during the malaria seasons between 2003 and 2005 to compare SP with AS plus SP, in order to provide evidence to health policy makers of further treatment options.

\section{Methods \\ Study location and design}

This multi-centre, open-label, parallel-group RCT was conducted in Maputo Province, Southern Mozambique in four public-sector health facilities in two phases: initially Catuane and Namaacha (2003), thereafter Boane and Magude (2004-2005) (Figure 1). There had been a marked decline in malaria prevalence throughout Maputo Province from 1999 until the time of the study start (Figure 1). This is attributed to an intensive intervention of indoor residual spraying (IRS) [11].

\section{Study subjects}

Male and non-pregnant, non-breastfeeding female patients with malaria symptoms, that were older than one year of age and weighed more than $10 \mathrm{~kg}$, were invited to give informed consent prior to screening for $P$. falciparum infection by rapid diagnostic test (Immunochromatographic Test, P.f ${ }^{\circledast}$, SA Scientific). Patients diagnosed with P. falciparum parasitaemia up to 500,000 asexual parasites/ $\mu \mathrm{ml}$ blood (on thick Giemsa-stained smear) and with axillary temperature greater than or equal to $37.5^{\circ} \mathrm{C}$ (or history of fever within the previous 24 hours) were suitable for inclusion. Patients receiving folate or antimalarials (including antibiotics with anti-malarial properties not used for malaria) within seven days were excluded. Also excluded were patients with danger signs (prostration, repeated vomiting and/or dehydration) or severely ill, those with a history of G6PD deficiency or allergy to the study (or related) drugs, or those with serious underlying disease $[12,13]$.

\section{Study treatments, enrolment and follow up}

Eligible subjects were randomized to treatment with SP monotherapy (Fansidar ${ }^{\circledR}$. Roche, Gauteng, South Africa) or AS/SP (co-packaged as Arsudar ${ }^{\circledR}$. Sanofi, Gauteng, South Africa) (Table 1). Subjects were observed until an hour post-dose in case a repeat dose was required for vomiting. A computerized random allocation schedule was generated for each site divided sequentially into the three weight-based dose levels, within which there was an equal chance of a subject receiving SP or AS/SP. Treatment allocation was concealed from site staff using opaque padded, sealed envelopes. Subsequently treatments were openlabel, except to microscopists who remained blinded to treatment allocation.

The visit schedule was based on the WHO protocol for assessment of in vivo therapeutic efficacy of anti-malarials, with extension to a 42 day follow-up due to the long elimination half-life of SP $[14,15]$. After enrolment on day 0 , subjects were asked to return on days $1,2,3,7,14,21,28$ 


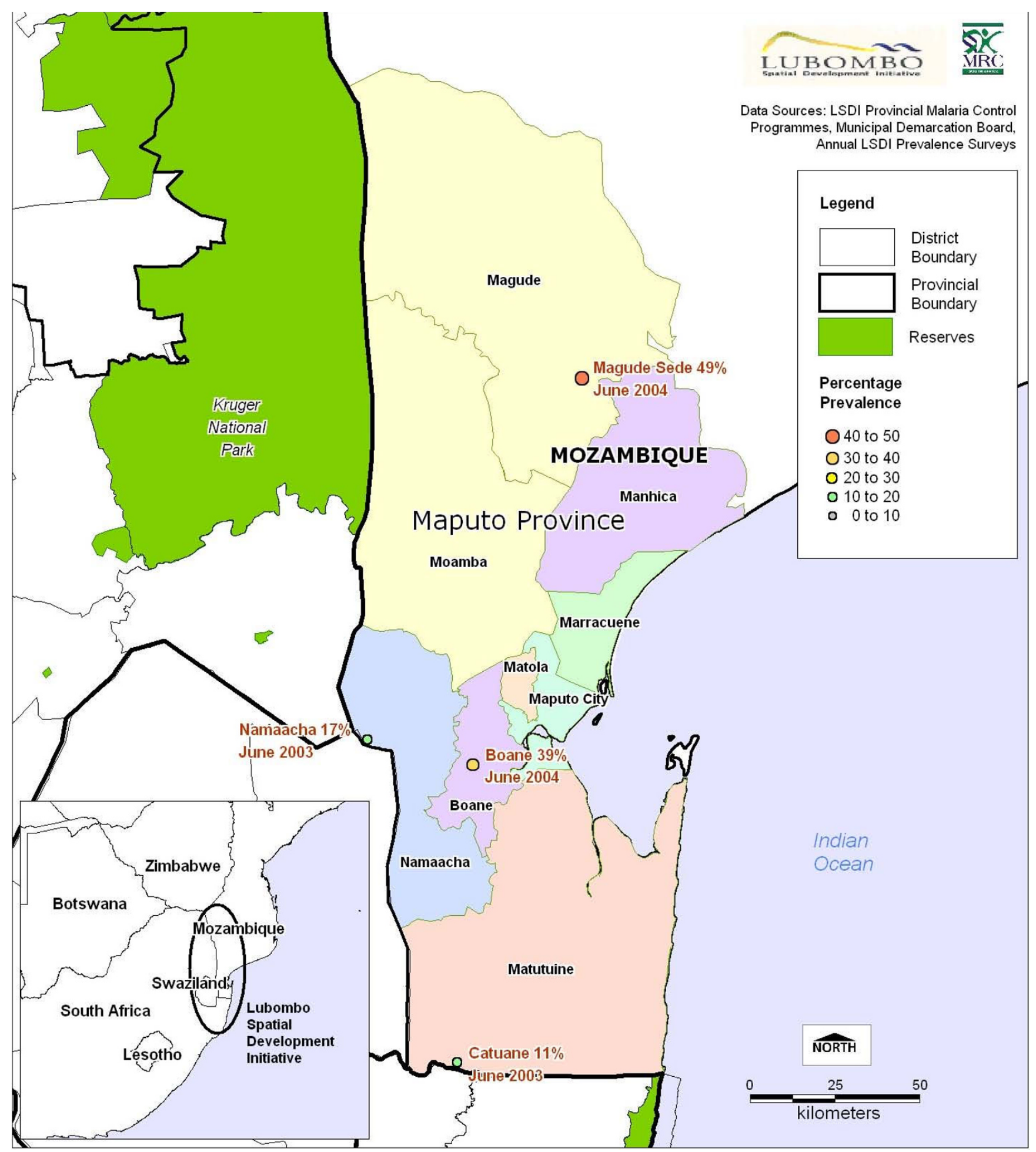

Figure I

Geographical location of the 4 study sites in Maputo Province showing prevalence of malaria at the study start. 
Table I: Numbers of artesunate $50 \mathrm{mg}$ tablets $(4 \mathrm{mg} / \mathrm{kg})$ and sulphadoxine-pyrimethamine $500 \mathrm{mg} / 25 \mathrm{mg}$ tablets $(25 / \mathrm{l} .25 \mathrm{mg} / \mathrm{kg}) \mathrm{for}$ each weight group

\begin{tabular}{|c|c|c|c|c|c|}
\hline \multirow[t]{2}{*}{ Mass $(\mathrm{kg})$} & \multirow[t]{2}{*}{ Approximate age (years) } & \multirow{2}{*}{$\begin{array}{l}\text { No. of SP tablets (all subjects) } \\
\text { Day } 0\end{array}$} & \multicolumn{3}{|c|}{ No. of AS tablets (AS/SP subjects) } \\
\hline & & & Day 0 & Day I & Day 2 \\
\hline $10-20$ & $1-6$ & I & I & I & I \\
\hline $21-35$ & $7-13$ & 2 & 2 & 2 & 2 \\
\hline$>35$ & $14+$ & 3 & 4 & 4 & 4 \\
\hline
\end{tabular}

SP: sulphadoxine-pyrimethamine

AS/SP: artesunate/sulphadoxine-pyrimethamine

and 42 for assessment of axillary temperature by electronic thermometer, asexual parasite density (by thick Giemsa-stained smear from finger-prick sample), haemoglobin concentration (by finger prick sample, HemoCue $^{\circledast}$ ), clinical signs and symptoms to capture adverse events, and concomitant medications [16]. A finger prick sample of blood blotted on filter paper (Whatman No. 1) was also taken at each visit for molecular analyses. Subjects were considered lost to follow up after three attempts to contact them by the study team.

\section{Laboratory investigations}

Asexual parasite density was calculated using the number of asexual parasites and assuming 8,000 leukocytes/ $\mu \mathrm{l}$ blood. Genotyping of P. falciparum DNA extracted from these dried samples based on variations in three highly variable proteins (merozoite surface proteins 1 and 2, and glutamine-rich protein) was used to determine if treatment failure was due to a re-infection or recrudescence of the original infection $[17,18]$. Infections were classified as recrudescent if PCR products for all three markers from day 0 and day of failure parasites were identical. If the banding patterns for any markers differed between day 0 and day of failure parasites, the infection was classified as a re-infection. Polymorphisms in the dihydrofolate reductase, dhfr (codons 51, 59, 108, 164) and dihydropteroate synthetase, dhps (codons 436, 437, 540 and 581) genes were detected using nested PCR and restriction endonuclease cleavage [19]. Digestion products separated on a $2 \%$ agarose gel using electrophoresis were visualized and photographed using a MiniBIS documentation system. Genotype of each codon was classified as pure wild, pure mutant or mixed (both mutant and wild alleles in one sample).

\section{Data management and analytical methods}

The primary study objective was to compare the risk of treatment failure between the treatment groups, adjusted for baseline characteristics. A secondary outcome compared the rate of parasite clearance between groups.
Response to treatment was classified according to WHO definitions for low to moderate malaria transmission intensity areas, modified for a 42-day follow up [14,15]. Subjects with clinical or parasitological failure, clinical deterioration other than treatment failure, an adverse event $(\mathrm{AE})$ requiring treatment withdrawal, allergic reaction during the treatment course, or concomitant prescribing of contra-indicated drugs, were withdrawn and given rescue treatment with quinine as necessary.

Key fields were verified by a study monitor and doubleentered into an MS Access 2000 (Microsoft Corporation, Seattle, USA) database. Laboratory data were recorded electronically. Data were imported into Stata/IC 10.0 (StataCorp LP, College Station, Texas, USA) when outcomes, time-to-event indicators and explanatory variables were programmed.

Major protocol violations were defined as subjects: i) missing days 1, 2, 3 of the ACT arm or days 2,3 of the monotherapy arm; ii) taking folic acid or a concomitant medication with anti-malarial activity (cotrimoxazole, trimethoprim, chloramphenicol and tetracyclines, with erythromycin added retrospectively to this list); iii), whose day 42 visit was more than three days late; and iv) taking an incorrect dose or repeat dose for vomiting. Data were not censored for missed visits as it was assumed intensive $\mathrm{AE}$ monitoring would detect malaria symptoms or treatment. Missing data fields were dropped where appropriate and subjects lost to follow-up after day 0 were automatically dropped from the time-to-event analyses. An attempt was made to follow all subjects to day 42 despite protocol violations.

Sample sizes were calculated assuming an adequate clinical and parasitological response (ACPR) of $75 \%$ for SP and $90 \%$ for AS/SP (confidence interval [CI] 95\% and $80 \%$ power) indicating 100 per treatment group in each phase of the study (400 subjects in total). 
Time-to-event outcomes, which were considered the most appropriate for the data, were analysed using Kaplan Meier (KM) survival methods and Cox's Proportional Hazards Regression. This allowed data up to the point of loss to follow-up or withdrawal to be included and for the relative hazard to approximate the relative risk.

KM distributions and survival curves for the two treatment groups were compared using a log rank test. Should model diagnostics suggest hazards were not proportional, parametric models were applied [20]. Time to treatment failure was defined as time from day 0 until day of failure due to recrudescence (early treatment failure [ETF], late clinical failure [LCF] or late parasitological failure [LPF]). Time to parasite clearance was time from day 0 to the first of two consecutive zero parasite density readings. The analyses were repeated after excluding subjects who had dose-related protocol violations and censoring the followup for all other protocol violations at the time of their occurrence.

\section{Ethical issues}

The study was approved by the Ethics Committees of the Mozambican Ministry of Health and the University of Cape Town prior to commencement, and conducted in accordance with the South African Clinical Trials Guidelines 2000 [21]. Staff members were trained in Good Clinical Practice including how to request informed consent in the local language. Illiterate patients marked ' $\mathrm{X}$ ' in the presence of an independent literate witness who signed the consent form. Any serious adverse events were reported to both ethics committees.

\section{Results}

Subject disposition is shown in Figure 2. Of 411 subjects randomized to SP or AS/SP, 359 (87.3\%) were followed to assessment of the study outcome (SP: 175 [87.9\%], AS/ SP 184 [86.8\%]). The main reason for loss to follow-up was reportedly movement from the study area, while one subject in each treatment group was withdrawn for an AE. There were no differences in baseline and clinical characteristics between treatment groups (Table 2) and protocol violations were also similar. Three subjects positive according to rapid diagnostic test and who completed the study were removed from all analyses as their day 0 slides were lost prior to parasite density confirmation. Of the remaining subjects, 27 (SP: 18 [10.3\%], AS/SP: 9 [4.9\%] p $=0.054)$ were rescued for re-treatment of malaria, but were found by PCR to have been re-infected. Excluding these re-infections, $138(88.5 \%)$ in the SP arm and 169 $(97.7 \%)$ in the AS plus SP arm achieved an ACPR (P = 0.0008 ). The proportion of ETFs was similar between the two treatment arms but there were significant differences for all other response categories, including re-infections (Table 3).

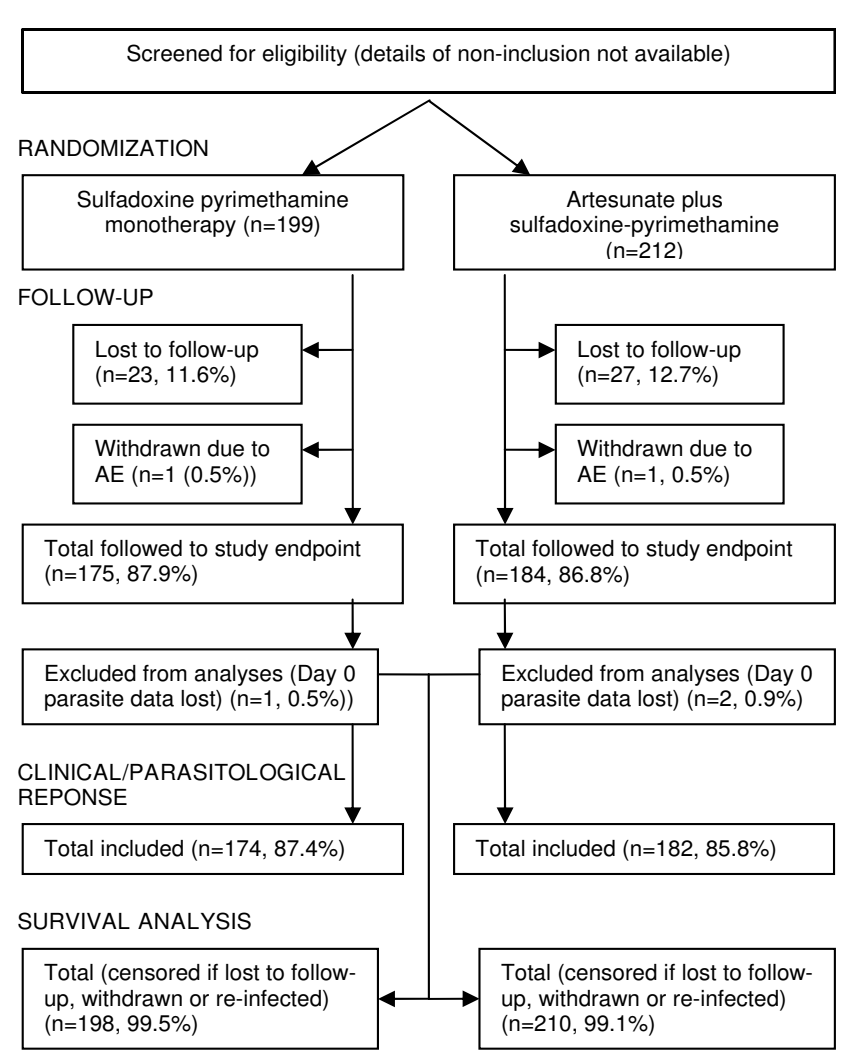

\section{Figure 2}

Subject disposition and the analysis dataset.

From the survival analysis, Figure 3 illustrates that ACPR rates were consistently higher in the ACT group compared to the SP monotherapy group (log-rank p-value = 0.0008 ). Day 42 success rates were $90.4 \%$ (95\% CI $84.9 \%-93.9 \%)$ for those taking SP monotherapy and 98.0\% (95\% CI 94.8\%-99.3\%) for those taking the ACT. The multivariate analysis (Table 4) showed the ACT decreased the relative hazard of failure by $80 \%$ compared to monotherapy, while age over seven years decreased the relative hazard of failure by $70 \%$ compared to younger age, baseline axillary temperature on Day 0 increased the relative hazard of failure by $50 \%$ for each additional $1{ }^{\circ} \mathrm{C}$, and a quintuple $\mathrm{dhfr} / \mathrm{dhps}$ mutation increased the relative hazard of failure 3.2 fold compared to fewer mutations. The proportional hazard assumption was satisfied overall; however there was an accelerated time to failure (approximately 6-fold) for subjects with a quintuple mutation compared to subjects with fewer mutations suggesting that early treatment failure is particularly associated with presence of a quintuple mutation. When the analysis was repeated after excluding or censoring for major protocol violations (subjects included; SP $n=177$ AS/SP $n=177$ ), results confirmed those found above. 
Table 2: Baseline and clinical characteristics of all subjects by treatment

\begin{tabular}{|c|c|c|c|c|}
\hline Continuous variables & $S P(n=199)$ & $\mathrm{AS} / \mathrm{SP}(\mathrm{n}=212)$ & Total $(n=4 I I)$ & P-value \\
\hline Haemoglobin $(\mathrm{g} / \mathrm{dL})($ mean/SD) & $11.02 .1)$ & $11.4(2.1)$ & $11.2(2.1)$ & $0.07 *$ \\
\hline Temperature $\left({ }^{\circ} \mathrm{C}\right)($ mean/SD) & $37.6(1.2)$ & $37.4(1.2)$ & $37.5(1.2)$ & $0.25 I^{*}$ \\
\hline Dose of pyrimethamine $(\mathrm{mg} / \mathrm{kg})(\mathrm{mean} / \mathrm{SD})$ & I.7(0.7) & $\mathrm{I} .8(0.6)$ & I.7 (0.6) & $0.645^{*}$ \\
\hline Dose of artesunate $(\mathrm{mg} / \mathrm{kg})($ mean $/ \mathrm{SD})$ & $\mathrm{N} / \mathrm{a}$ & $11.4(3.0)$ & $11.4(3.0)$ & $\mathrm{N} / \mathrm{a}$ \\
\hline Age (years) (median/lQR) & $10.0(5.0-22.0)$ & $12.0(5.0-24.0)$ & $11.0(5.0-23.0)$ & $0.237 \#$ \\
\hline Weight (kg) (median/IQR) & $25.0(15.0-52.0)$ & $28.0(15.0-55.0)$ & $25.8(15.0-54.0)$ & $0.508 \#$ \\
\hline Parasite density $($ per $\mu \mathrm{l})$ (geometric mean/95\% Cl) & $\begin{array}{c}3630.7 \\
(2464.8 ; 5348.1)\end{array}$ & $\begin{array}{c}2885.2 \\
(2028.4 ; 4104.0)\end{array}$ & $\begin{array}{c}3225.6 \\
(2486.3 ; 4 \mid 84.8)\end{array}$ & $0.284 \#$ \\
\hline Log parasite density (per $\mu \mathrm{l})$ (median/IQR) & $3.8(2.5-4.5)$ & $3.6(2.5-4.5)$ & $3.7(2.5-4.5)$ & $0.315 \#$ \\
\hline Duration malaria symptoms (days) (median/IQR) & $1.0(1.0-3.0)$ & $2.0(1.0-3.0)$ & $2.0(1.0-3.0)$ & $0.347 \#$ \\
\hline \multicolumn{5}{|l|}{ Categorical variables (n/\%) } \\
\hline Gender male & $76(38)$ & $94(44)$ & $170(4 \mid)$ & $0.206^{\wedge}$ \\
\hline Age 7 years or under & $84(42.2)$ & $79(37.3)$ & $163(39.7)$ & $0.306^{\wedge}$ \\
\hline Anaemic (haemoglobin < II g/dL)! & $94(47)$ & $94(44)$ & $188(46)$ & $0.556^{\wedge}$ \\
\hline Severely anaemia (haemoglobin $<7 \mathrm{~g} / \mathrm{dL}$ ) & $6(3)$ & $2(1)$ & $8(2)$ & $0.129^{\wedge}$ \\
\hline History of vomiting & $20(10)$ & $17(8)$ & $37(9)$ & $0.472^{\wedge}$ \\
\hline History of vomiting, with fever on day 0 & $9(5)$ & $6(3)$ & $15(4)$ & $0.361^{\wedge}$ \\
\hline Vomited within I hour of any dose & $7(4)$ & $12(6)$ & $19(5)$ & $0.301^{\wedge}$ \\
\hline Diarrhoea in first $24 \mathrm{hrs}$ post dose & $14(7)$ & $7(3)$ & $21(5)$ & $0.086^{\wedge}$ \\
\hline Diarrhoea between days 2 and 7 & $7(4)$ & $5(2)$ & $12(3)$ & $0.485^{\wedge}$ \\
\hline Quintuple mutation & $45 / 195(23.1)$ & $39 / 210(18.6)$ & $84(20)$ & $0.289^{\wedge}$ \\
\hline
\end{tabular}

SP: sulphadoxine-pyrimethamine

AS/SP: artesunate/sulphadoxine-pyrimethamine

I WHO 200I

N/a Not applicable

* Student's two sample t-tests

\#Wilcoxon rank sum test

$\wedge$ Chi-square or Fisher's exact tests

Table 3: Clinical and parasitological response by treatment group for subjects completing follow up

\begin{tabular}{lllll}
\hline $\begin{array}{l}\text { Response } \\
n(\%)\end{array}$ & $\begin{array}{l}S P \\
N=174\end{array}$ & $\begin{array}{l}\text { AS/SP } \\
N=182\end{array}$ & $\begin{array}{l}\text { Total } \\
N=356\end{array}$ & $P^{*}$ \\
\hline Re-infection & $18(10.3)$ & $9(4.9)$ & $27(7.6)$ & 0.054 \\
\hline Success (ACPR) & $138(88.5)$ & $169(97.7)$ & $307(93.3)$ & 0.0008 \\
Treatment failure & $18(11.5)$ & $4(2.3)$ & $22(6.7)$ & 0.0008 \\
$\quad$ ETF & $5(3.2)$ & $3(1.7)$ & $8(2.4)$ & 0.39 \\
LCF & $4(2.6)$ & 0 & $4(1.2)$ & 0.03 \\
LPF & $9(5.8)$ & $1(0.6)$ & $10(3.0)$ & 0.006
\end{tabular}

As patients with re-infection were withdrawn and rescued prior to study completion they are excluded form denominators for ACPR and treatment failure

SP: sulphadoxine-pyrimethamine

AS/SP: artesunate/sulphadoxine-pyrimethamine

ACPR: adequate clinical and parasitological response

ETF: early treatment failure

LCF: late clinical failure

LPF: late parasitological failure

*2-sample test of proportions

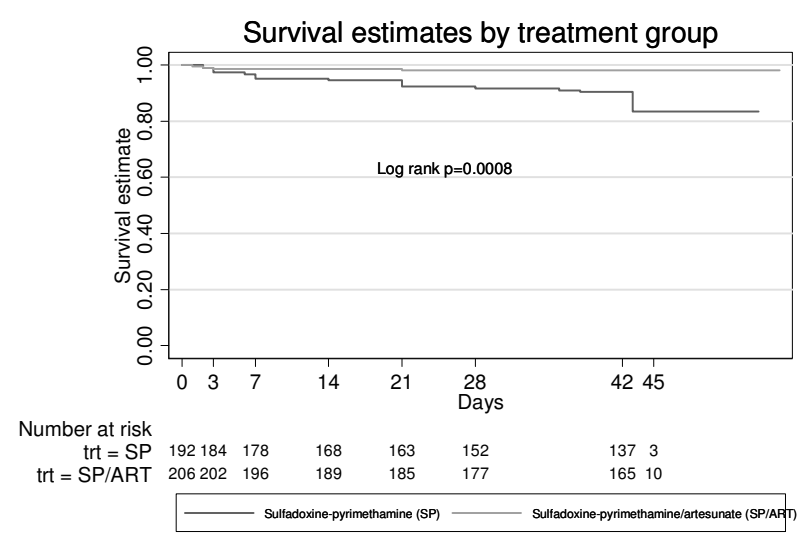

Figure 3

Kaplan-Meier survival curves for time to treatment failure. 
Table 4: Multivariate model for the relative risk of treatment failure

\begin{tabular}{lll}
\hline Variable & Hazard ratio & $95 \% \mathrm{Cl}$ \\
\hline Treatment with AS/SP vs SP & 0.2 & $0.1-0.6$ \\
Age $>7$ years vs. age $\leq 7$ years & 0.3 & 0.004 \\
Presence of a quintuple mutation vs. fewer mutations & 3.2 & 0.033 \\
Temperature Day 0 $\left({ }^{\circ} \mathrm{C}\right)$ & 1.5 & 0.009 \\
\hline
\end{tabular}

SP: sulphadoxine-pyrimethamine

AS/SP: artesunate/sulphadoxine-pyrimethamine

The ACT group had faster parasite clearance rates compared to the SP monotherapy group (log-rank $\mathrm{p}=0.001$ ) (Figure 4). Multivariate analysis (Table 5) found that ACT increased the rate of parasite clearance by $80 \%$ compared with monotherapy. In addition each 10 -fold increase in baseline parasite density decreased parasite clearance by $20 \%$. Subjects from Catuane cleared parasites $80 \%$ quicker compared to those from other sites. A significant interaction between treatment and parasite density suggested a faster parasite clearance by the ACT compared to monotherapy at higher parasite densities (HR 1.2; 95\% CI $1.0-1.5 \mathrm{p}=0.033$ ). However, the relative hazard of clearing parasites was similar for both treatment groups when modelled separately, indicating our overall results hold true for all subjects. The proportional hazard assumption was satisfied overall and for the treatment and Catuane effects. The baseline parasite density, however, was found to amplify the parasite clearance time by approximately $30 \%$ for every 10 -fold increase. When the analysis was repeated excluding subjects with dose-related protocol violations, or censoring at the time of other violations, results were similar although there were no interactions identified, and age over seven years (HR 1.3: 95\% CI 1.0$1.7 \mathrm{p}=0.046)$ was predictive of better parasite clearance compared to younger age.

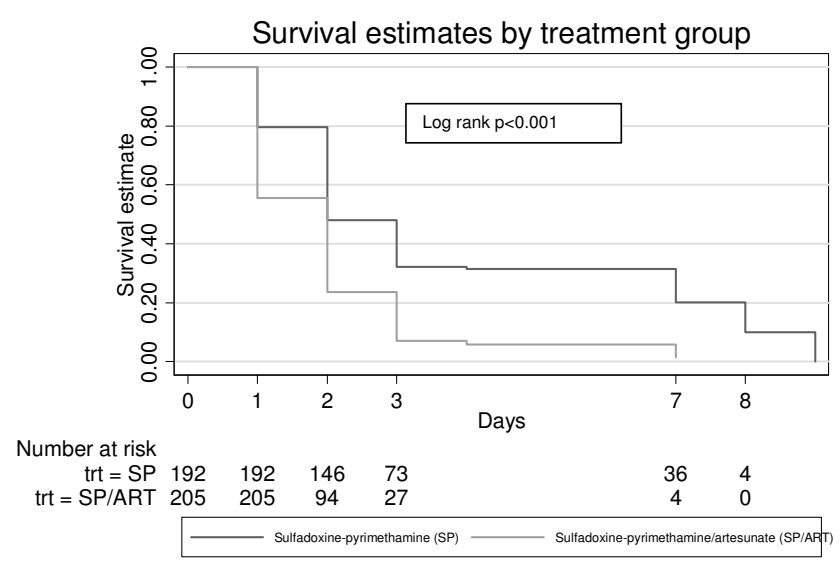

\section{Figure 4}

Kaplan-Meier survival curves for time to parasite clearance.

\section{Discussion}

The aim of this study was to provide evidence to Mozambican health policy-makers regarding efficacy of SP with or without AS as treatment for uncomplicated malaria. In a survival analysis, both drug regimens were effective, however adding artesunate improved outcomes significantly with regard to clinical and parasitological cure, and peripheral parasite clearance time. These results are consistent with the literature, that suggests combining AS with SP improves efficacy, provided efficacy of SP is sufficiently high [7]. In addition, AS combined with SP showed an efficacy above 95\%, the minimum level recommended by the WHO for policy implementation. The increased parasite clearance by the ACT corroborates data reflecting the higher parasite reduction ratio of the artemisinins (approximately $10^{3}-10^{5}$ parasites per asexual life cycle, compared to $10-10^{3}$ for SP) [3].

Subjects aged over seven years had a 70\% lower risk of treatment failure than those seven and under, and were $30 \%$ more likely to clear parasites faster (the latter association was only found when protocol violators were removed). These findings are consistent with the knowledge that immunity to malaria infection increases with age [22]. However, this protective effect is not fully understood as there is no clear immunological marker with which to validate it, and risk factors for malaria are entwined due to the vector, host and parasite relationships [22-24]. Another possible explanation for associations between lower age and poorer treatment outcome is that drug disposition in children may be altered leading to concentrations below the therapeutic threshold; physiological concentrations of anti-malarials at $\mathrm{mg} / \mathrm{kg}$ labelled doses, particularly SP, may be sub-optimal in younger patients $[25,26]$. Dosing regimens derived from studies recruiting adults may lead to inappropriate recommendations for children particularly if regimens are age-rather than weight-based, although the former is more practical in resource-poor environments [27]. Conversely, adults may be under-dosed if they weigh more than $65 \mathrm{~kg}$ [28]. Bearing these findings in mind, healthcare providers should be trained to monitor children closely, in particular, for treatment failure. 
Table 5: Multivariate model for the relative risk of parasite clearance

\begin{tabular}{llll}
\hline Variable & Hazard ratio & $95 \%$ CI & $P$ \\
\hline Treatment with AS/SP vs. SP & 1.8 & $1.4-2.3$ & $<0.001$ \\
Log 10 parasite density (per $\mu$ l) blood) & 0.8 & $0.7-0.8$ & $<0.001$ \\
Catuane (compared to other sites) & 1.8 & $1.3-2.5$ & $<0.001$ \\
\hline
\end{tabular}

SP: sulphadoxine-pyrimethamine

AS/SP: artesunate/sulphadoxine-pyrimethamine

Sulphadoxine and pyrimethamine are anti-folates that inhibit the dihydropteroate synthetase (dhps) and dihydrofolate reductase $(d h f r)$ enzymes respectively. Resistance to SP develops due to accumulation of point mutations in genes that encode these enzymes. The presence of 5 mutations, 3 in the dhfr gene (S108N/N511/ $\mathrm{C} 59 \mathrm{R})$ and 2 in the dhps gene (A437G/K540E) are associated with treatment failure $[29,30]$. This study found presence of the quintuple mutation was associated with a more than 3-fold increase in relative hazard of treatment failure compared to fewer mutations. Similar observations elsewhere have motivated proposals that molecular markers of resistance be used to predict future therapeutic response at the population level due to the myriad factors playing a role in treatment response [31]. The unexpected improvement in SP monotherapy cure rates between 2002, and this study's findings, may be explained in the decreased frequency of quintuple mutations seen in this province after the replacement of SP monotherapy with artemether-lumefantrine policy in neighbouring KwaZulu-Natal (KZN) [10]. However, this mutation frequency is expected to have increased again markedly since the study was concluded as SP containing regimens have been implemented country-wide for both the $1^{\text {st }}$ line treatment of uncomplicated malaria (AQ-SP replaced with AS$\mathrm{SP})$ and as intermittent preventive treatment for pregnant women (SP in 2007).

This study showed a significant association between higher temperature at baseline and greater relative hazard of treatment failure, as found in other studies [32]. The biological mechanism for this association is unclear, and temperature fluctuations, the concomitant use of pyretics for malaria and the presence other febrile conditions all indicates body temperature is an inconsistent predictor of outcome.

Higher parasitaemia at baseline was not found to be an independent risk factor for treatment failure, as opposed to findings in other studies, although it was associated with delayed parasite clearance [33]. The interaction observed between treatment and parasite density suggested the ACT effect was amplified at higher parasitaemias, as would be expected given the specific indication for artemisinin derivatives in uncomplicated hyperparasi- taemias [34]. However, this interaction did not translate into a clear difference between treatments.

This study was able to achieve reasonably good subject retention and completeness of data in resource-poor community settings, which have inherent migration risk factors. Standardized training and methodologies for outcomes' measurements together with quality control measures, especially those relating to parasitological end points, minimized the possibility of measurement error. While the study was open-label after concealed allocation, the primary end point was measured by staff blinded to treatment group. Recrudescence occurred beyond 42 days, albeit in small numbers, validating this minimum recommended schedule and suggesting that, should resources allow, follow up be extended further [35].

When data were re-analysed excluding 57 (13.9\%) protocol violators, results were very similar. Including data from non-adherent subjects or those taking prohibited concomitant medications is useful for assessing the effect of these common practices on treatment response. AS courses shorter than three days are not recommended due to their lower efficacy and the increased likelihood of antimalarial drug resistance emerging if combined with the more slowly eliminated SP [5,6]. SP may, however, be sufficient to successfully treat malaria in individual subjects who take a substantially reduced dose of AS should its own efficacy be high enough. The study showed that in $90 \%$ of subjects SP monotherapy was able to effect an ACPR. The retrospective addition of erythromycin as a prohibited drug meant it was the predominant contributor to this subset of violations (36/43 [83.7\%]), however there was no evidence of erythromycin playing a role in curing uncomplicated malaria or improving parasite clearance.

\section{Conclusion}

This study found that, while both SP monotherapy and in combination with AS were effective, the ACT was far superior, supporting its current use in Mozambique as the national policy for the treatment of uncomplicated malaria. However, it is recognized that combinations with SP may have a limited useful life due to the spread of parasite resistance, and inadequate drug levels young chil- 
dren achieve. It is also possible that SP efficacy has declined since this study due to the national implementation of SP as an intermittent preventive treatment policy for pregnant women during 2007. Of further concern is the presence of the $d h f r 164$ mutation, associated with high-level pyrimethamine resistance found in neighbouring Malawi [36].

\section{Competing interests}

The authors declare that they have no competing interests.

\section{Authors' contributions}

EA participated in study design, coordinated the study, conducted the statistical analysis and drafted the manuscript, TC managed the Mozambique study teams, YC provided medical expertise to the study teams, FL participated in study design, calculated sample sizes and supervised the statistical analysis, JR conducted molecular analyses, AB supervised analysis and reporting. KIB, Principal Investigator of the South East African Combination Anti-malarial Treatment (SEACAT) Evaluation, conceived of, and designed the study, and contributed to the analysis. All authors read and approved the final manuscript.

\section{Acknowledgements}

Many thanks to the patients and staff at the participating health facilities, and to Amanda Jackson's team at the Malaria Lead Programme, Medical Research Council, Durban for data entry. The authors wish to acknowledge the financial support provided by the UNDP/World Bank/WHO Special Programme for Research and Training in Tropical Diseases (TDR) to the South East African Combination Antimalarial Therapy (SEACAT) Evaluation and the funding of the Lebombo Spatial Development Initiative in Maputo Province by the Global Fund for fighting AIDS, tuberculosis and malaria.

\section{References}

I. World Health Organization, Regional Office for Africa. Country health system fact sheet 2006 Mozambique [http:// www.who.int/countries/moz/moz/en/]

2. Wellems TE, Plowe CV: Chloroquine-resistant malaria. J Infect Dis 200I, 184:770-76.

3. White NJ: Assessment of the pharmacodynamic properties of antimalarial drugs in vivo. Minireview. Antimicrob Agents Chemother 1997, 4 I: | 413-22.

4. Price RN, Nosten F, Luxemburger C, ter Kuile FO, Paiphun L, Chongsuphajaisiddhi T, White NJ: Effects of artemisinin derivatives on malaria transmissibility. Lancet 1996, 347: 1654-8.

5. Nosten F, White NJ: Artemisinin-based combination treatment of falciparum malaria. Am J Trop Med Hyg 2007, 77(Suppl 6): $|8|-92$.

6. von Seidlein L, Milligan P, Pinder M, Bojang K, Anyalebech C, Gosling $R$ : Efficacy of artesunate plus pyrimethamine-sulphadoxine for uncomplicated malaria in Gambian children: a doubleblind, randomised, controlled trial. Lancet 2000, 355:352-57.

7. World Health Organization: Guidelines for the treatment of malaria

TreatmentGuidelines2006.pdf]

8. International Artemisinin Study Group: Artesunate combinations for treatment of malaria: meta-analysis. Lancet 2004, 363:9-I7.

9. WorldWide Antimalarial Resistance Network (WARN) [http://www.wwarn.org/home/index.php]

10. Raman J, Sharp B, Kleinschmidt I, Roper C, Streat E, Kelly V, Barnes $\mathrm{KI}$ : Differential effect of regional drug pressure on dihydrofolate reductase and dihydropteroate synthetase mutations in southern Mozambique. Am J Trop Med Hyg. 2008, 78(2):256-26I.

II. Sharp BL, Kleinschmidt I, Streat E, Maharaj R, Barnes KI, Durrheim DN: Seven years of regional malaria control collaborationMozambique, South Africa and Swaziland. Am J Trop Med Hyg. 2007, 76(I):42-47.

12. Carter JY, Loolpapit MP, Lema OE, Tome JL, Nagelkerke NJD, Watkins WM: Reduction of the efficacy of antifolate antimalarial therapy by folic acid supplementation. Am J Trop Med Hyg 2005, 73: $166-70$.

13. World Health Organization: Severe falciparum malaria. Trans $R$ Soc Trop Med Hyg 2000, 94(Suppl I):62

14. World Health Organization: Assessment of therapeutic efficacy of antimalarial drugs for uncomplicated falciparum malaria in areas with intense transmission. WHOIMAU96.1077 1996.

15. World Health Organization, Assessment and monitoring of antimalarial drug efficacy for the treatment of uncomplicated falciparum malaria [http://www.who.int/malaria/docs/Pro tocolWHO.pdf]

16. World Health Organization: Basic malaria microscopy Part I Learner's Guide Switzerland: Macmillan/Presses Centrales; I99I.

17. Wooden J, Keyes S, Sibley CH: PCR and strain identification in Plasmodium falciparum. Parasitol Today 1993:303-5.

18. Ranford-Cartwright LC, Taylor J, Umasunthar T, Taylor LH, Babiker HA, Lell B, Schmidt-Ott JR, Lehman LG, Walliker D, Kremsner PG: Molecular analysis of recrudescent parasites in a Plasmodium falciparum drug efficacy trial in Gabon. Trans $R$ Soc Trop Med Hyg. 1997, $91(6): 719-724$.

19. Plowe CV, Cortese JF, Djimde A, Nwanyanwu OC, Watkins WM, Winstanley PA, Estrada-Franco JG, Mollinedo RE, Avila JC, Cespedes JL, Carter D, Doumbo OK: Mutations in Plasmodium falciparum dihydrofolate reductase and dihydropteroate synthase and epidemiologic patterns of pyrimethamine-sulfadoxine use and resistance. J Infect Dis 1997, I 76:1590-6.

20. Collett D: Modelling survival data in medical research 2 nd edition. Chapman and Hall/CRC. Press LLC; 2003.

21. Department of Health: Guidelines for good practice in the conduct of clinical trails in South Africa Department of Health, Pretoria, South Africa; 2000.

22. Haviid L: Naturally acquired immunity to Plasmodium falciparum malaria in Africa. Acta Trop Acta Trop. 2005, 95(3):270-275.

23. Aponte JJ, Menendez C, Schellenberg D, Kahigwa E, Mshinda H, Vountasou P, Tanner M, Alonso PL: Age interactions in the development of naturally acquired immunity to Plasmodium falciparum and its clinical presentation. PLoS Med. 2007, 4(7):e242.

24. Dorsey G, Kamya MR, Ndeezi G, Babirye JN, Phares CR, Olson JE, Katabira ET, Rosenthal PJ: Predictors of chloroquine treatment failure in children and adults with falciparum malaria in Kampala, Uganda. Am J Trop Med Hyg. 2000, 62(6):686-692.

25. Barnes KI, Little F, Smith PJ, Evans A, Watkins WM, White NJ: Sulfadoxine-pyrimethamine pharmacokinetics in malaria: pediatric dosing implications. Clin Pharmacol Ther. 2006, 80(6): $582-596$.

26. Barnes $\mathrm{KI}$, Watkins WM, White NJ: Antimalarial dosing regimens and drug resistance. Trends Parasitol. 2008, 24(3): I27-I34.

27. Terlouw DJ, Courval JM, Kolczak MS, Rosenberg OS, Oloo AJ, Kager PA, Lal AA, Nahlen BL, ter Kuile FO: Treatment history and treatment dose are important determinants of sulfadoxinepyrimethamine efficacy in children with uncomplicated malaria in Western Kenya. J Infect Dis 2003, I 87:467-76.

28. Govere JM, la Grange JJ, Durrheim DN, Freese JA, Sharp BL, Mabuza A, Mngomezulu N, Bredenkamp BL: Sulfadoxine-pyrimethamine effectiveness against Plasmodium falciparum malaria in Mpumalanga Province, South Africa. Trans R Soc Trop Med Hyg 1999, 93:644.

29. Plowe CV, Cortese JF, Djimde A, Nwanyanwu OC, Watkins WM, Winstanley PA, Estrada-Franco JG, Mollinedo RE, Avila JC, Cespedes $J \mathrm{~L}$, Carter D, Doumbo OK: Mutations in Plasmodium falciparum dihydrofolate reductase and dihydropteroate synthase and epidemiologic patterns of pyrimethamine-sulfadoxine use and resistance. J Infect Dis 1997, I 76(Suppl 6): I590-6.

30. Kublin JG, Dzinjalamala FK, Kamwendo DD, Malkin EM, Cortese JF, Martino LM, Mukadam RA, Rogerson SJ, Lescano AG, Molyneux ME, Winstanley PA, Chimpeni P, Taylor TE, Plowe CV: Molecular mark- 
ers for failure of sulfadoxine-pyrimethamine and chlorproguanil-dapsone treatment of Plasmodium falciparum malaria. J Infect Dis 2002, I 85(Suppl 3):380-8.

31. Plowe CV, Roper C, Barnwell JW, Happi CT, Joshi HH, Mbacham W, Meshnick SR, Mugittu K, Naidoo I, Price RN, Shafer RW, Sibley CH, Sutherland C], Zimmerman PA, Rosenthal PJ: World Antimalarial Resistance Network (WARN) III: molecular markers for drug resistant malaria. Malar J 2007, 6:121.

32. Hamer DH, MacLeod WB, Addo-Yobo E, Duggan CP, Estrella B, Fawzi WW, Konde-Lule JK, Mwanakasale V, Premji ZG, Sempértegui F, Ssengooba FP, Yeboah-Antwi K, Simon JL: Age, temperature, and parasitaemia predict chloroquine treatment failure and anaemia in children with uncomplicated Plasmodium falciparum malaria. Trans $R$ Soc Trop Med Hyg 2003, 97(Suppl 4):422-8.

33. Fontanet $A L$, Walker $A M$ : Predictors of treatment failure in multiple drug resistant falciparum malaria: results from a 42 day follow-up of 224 patients in Eastern Thailand. Am J Trop Med Hyg 1993, 49(4):465-72.

34. Luxemburger C, Nosten F, Raimond SD, Chongsuphajaisiddhi T, White NJ: Oral artesunate in the treatment of uncomplicated hyperparasitemic falciparum malaria. Am J Trop Med Hyg. 1995, 53(5):522-525.

35. Stepniewska K, Taylor WR, Mayxay M, Price R, Smithuis F, Guthmann $J P$, Barnes K, Myint HY, Adjuik M, Olliaro P, Pukrittayakamee S, Looareesuwan S, Hien TT, Farrar J, Nosten F, Day NP, White NJ: In vivo assessment of drug efficacy against plasmodium falciparum malaria: duration of follow-up. Agents Chemother. 2004, 48(I I):427I-4280.

36. Alker AP, Mwapasa V, Purfield A, Rogerson SJ, Molyneux ME, Kamwendo DD, Tadesse E, Chaluluka E, Meshnick SR: Mutations associated with sulfadoxine-pyrimethamine and chlorproguanil resistance in Plasmodium falciparum isolates from Blantyre, Malawi. Antimicrob Agents Chemother. 2005, 49(9):3919-3921.

Publish with Bio Med Central and every scientist can read your work free of charge

"BioMed Central will be the most significant development for disseminating the results of biomedical research in our lifetime. "

Sir Paul Nurse, Cancer Research UK

Your research papers will be:

- available free of charge to the entire biomedical community

- peer reviewed and published immediately upon acceptance

- cited in PubMed and archived on PubMed Central

- yours - you keep the copyright

Submit your manuscript here:

http://www.biomedcentral.com/info/publishing_adv.asp
BiolMedcentral 